\title{
Fluid front morphologies in gap-modulated Hele-Shaw cells
}

\author{
Lautaro Díaz-Piola, ${ }^{1}$ Ramon Planet, ${ }^{1,2}$ Otger Campàs, ${ }^{3}$ Jaume Casademunt, ${ }^{1,2}$ and Jordi Ortín ${ }^{1,2, *}$ \\ ${ }^{1}$ Departament de Física de la Matèria Condensada, Universitat de Barcelona, Martí i Franquès 1, \\ 08028 Barcelona, Spain \\ ${ }^{2}$ Universitat de Barcelona Institute of Complex Systems, Barcelona, Spain \\ ${ }^{3}$ Department of Mechanical Engineering, and California NanoSystems Institute, University of California, \\ Santa Barbara, California 93106, USA
}

(Received 26 May 2017; published 25 September 2017)

\begin{abstract}
We consider the displacement of an inviscid fluid (air) by a viscous fluid (oil) in a narrow channel with gap-thickness modulations. The interfacial dynamics of this problem is strongly nonlocal and exhibits competing effects from capillarity and permeability. We derive analytical predictions of steady-state front morphologies, which are exact at linear level in the case of a persistent modulation in the direction of front advancement. The theoretical predictions are in good agreement with experimental measurements of steadystate front morphologies obtained in a Hele-Shaw cell with modulations of the channel depth, consisting on three parallel tracks of reduced depth, for small gap modulations. The relative average distance between theoretical and experimental fronts in the region around the central track is smaller than about $4 \%$, provided that the height of the tracks is less than $13 \%$ of the total channel depth and the local distortion of the front height $h$ is small enough $(|\nabla h|<0.8)$ for the linear approximation to hold.
\end{abstract}

DOI: 10.1103/PhysRevFluids.2.094006

\section{INTRODUCTION}

The study of controlled spatiotemporal modulations in problems of pattern formation is a very useful conceptual tool to elucidate dynamic features which may not be easily accessible in realistic situations [1]. Patterned channels in particular appear in many problems involving multiphase flows, such as mixing in microfluidic devices [2], droplet manipulation [3,4], and flow in disordered media $[5,6]$. In these examples simple modifications of surface topography are sufficient to qualitatively alter the fluid dynamics.

On the other hand, the case study of fluid-fluid interface dynamics in Hele-Shaw cells is a paradigmatic model system that has consistently provided useful insights into mechanisms that may be generic in a broad variety of systems in interfacial pattern formation [7]. In this context, gap-modulated Hele-Shaw cells have been studied previously as a means of controlling the SaffmanTaylor viscous fingering instability that takes place when a more viscous fluid is displaced by a less viscous fluid [8-10] and of perturbing the resulting Saffman-Taylor stationary finger $[11,12]$.

While the reverse displacement of a less viscous fluid by a more viscous fluid is clearly a simpler problem, the presence of gap-thickness modulations introduces local fluctuations of capillary pressure and permeability in an otherwise stable situation. For this reason Hele-Shaw cells featuring spatially quenched disorder in the form of randomly placed obstacles [13,14] or gap-thickness modulations $[5,6,15]$ have been useful to study fluid imbibition in disordered media (porous materials and open fractures, respectively; see Ref. [16] for a review). Stable imbibition is a technologically important fluid-fluid displacement present in soil irrigation, printing, filtering, and oil recovery, for example.

The dynamics of stable imbibition fronts has also attracted attention from a fundamental point of view, in the context of nonequilibrium driven interfaces. Competition between distorting forces, generated by the spatially quenched disorder, and the stabilizing forces of viscous pressure and

*jordi.ortin@ub.edu 
interfacial tension, acting on different scales, gives rise to complex (scale-invariant) interfacial morphologies $[5,13,15]$ and intermittent spatiotemporal dynamics $[14,17,18]$.

In contrast to many other interfacial problems, the interfacial dynamics in Hele-Shaw flows is strongly nonlocal [6,19-21]. To gain understanding on how local disturbances affect the overall interface shape with such nonlocal dynamics, here we study a stable imbibition displacement in which a viscous and wetting fluid (oil) displaces a nearly inviscid and less wetting fluid (air) in a rectangular Hele-Shaw cell with a persistent modulation of the channel depth in the direction transverse to the direction of front advancement. The modulation is generated by a topographic relief of the bottom plate of the cell.

Competing effects in stable imbibition displacements in disordered media have been studied extensively, but the simpler problem addressed here of how a regular modulation of the medium deforms the interface has not been studied before with the present level of accuracy. De Gennes pioneered theoretical efforts to understand the morphology and dynamics of an otherwise stable fluid meniscus in a Hele-Shaw cell with gap-thickness modulations [19]. As he pointed out, a clear understanding of the influence of a simple modulation on the interfacial dynamics is a prerequisite to understand the complexity of stable imbibition displacements in disordered media.

We describe the interfacial dynamics theoretically within a linear approximation and characterize how spatially persistent variations in gap thickness affect the steady-state morphology of the advancing fluid-air interface [22]. We show that while capillary effects produced by the variation in gap thickness induce local deformations of the interface, permeability contributions have a long range, unbounded effect on the interface. The predictions of our linear theoretical model are compared to experimental data of stably advancing fronts in a Hele-Shaw cell with controlled and persistent depth modulations, for varying gap thickness and advancing front velocity. Our results show that the theoretical results properly account for the experimental observations in the limit of moderate interfacial deformations and small gap gradients.

We hope that our results will provide insight into the basic mechanisms underlying the spatiotemporal dynamics of stable fluid invasion of disordered media [16] and will be relevant also for studies of interfacial phenomena in confined geometries, such as flows in microfluidic channels [23], where topography finds its use as a passive mechanism to generate recirculation in the cross-section of steady, pressure-driven flows [2], and controlled drop breakup [3,4].

The paper is organized as follows. The theoretical framework is presented in Sec.II, and the experimental setup in Sec.III. Experimental results and comparison to theoretical predictions are presented in Sec.IV. In Sec.V we discuss the limits of our theoretical approach and compare our results with other interfacial problems. The main conclusions of our work are finally drawn in Sec.VI.

\section{THEORETICAL FRAMEWORK}

The interfacial dynamics of an imbibition front in a Hele-Shaw cell of variable gap thickness has been previously derived ab initio [22,24]. Local variations in gap thickness lead to two distinct physical effects, namely, variations in the local capillary pressure at the interface and variations in the local permeability of the medium, both affecting the morphology and dynamics of the fluid front. In spite of having a common geometrical origin, these two effects are different and often counteracting. As shown in this section, the dynamical response of the front to external disturbances in the form of gap-thickness variations is a complex problem already at linear level, because of the interplay between capillary and permeability effects and the strongly nonlocal character of the front morphology.

We consider a two-phase displacement, in which a viscous fluid (e.g., oil) invades a horizontal Hele-Shaw cell and displaces an inviscid fluid of negligible viscosity (e.g., air) at constant flow rate (Fig. 1). $V$ is the constant velocity of the incoming fluid at the inlet, away from the interface. We define a spatial modulation in gap thickness, $\zeta(x, y)$, through

$$
b^{2}(x, y)=b_{0}^{2}[1+\zeta(x, y)]
$$




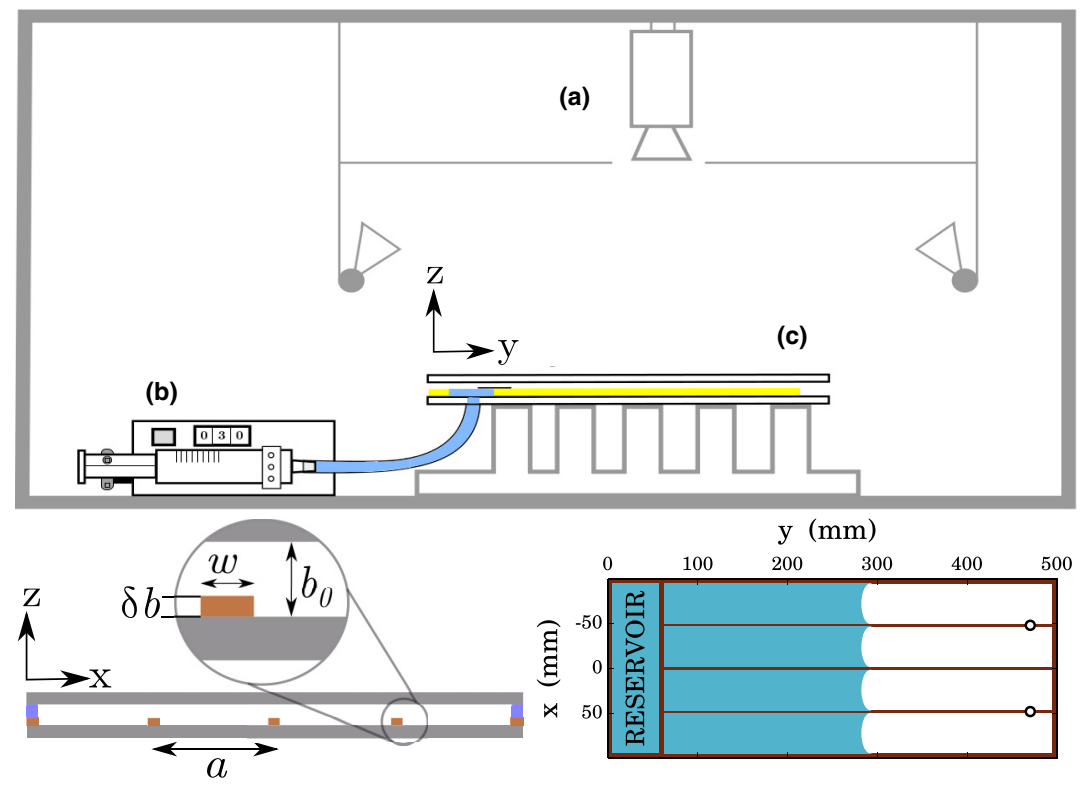

FIG. 1. Top: Basic representation of the experimental setup. (a) Image acquisition system; (b) syringe pump; (c) Hele-Shaw cell. Bottom: (Left) Cross section of the gap-modulated Hele-Shaw cell. (Right) Top view of the Hele-Shaw cell. The oil, represented in blue, displaces the air present in the cell, and the air flows out of it through two holes at the end of the top plate.

where $b(x, y)$ is the gap thickness at $(x, y)$ and $b_{0}$ is the homogeneous gap thickness in the absence of topographic relief. Assuming that the modulation in gap thickness is smooth enough, i.e., $|\nabla b| \ll 1$, Darcy's law for a Hele-Shaw cell can be considered locally valid:

$$
\vec{v}=-\frac{b^{2}(x, y)}{12 \mu} \nabla p
$$

where $\vec{v}$ is the two-dimensional velocity field and $\nabla p$ is the local pressure gradient. The assumption of a local Darcy flow allows to derive the interfacial equation in terms of the spatial dependence of the gap thickness without the necessity of coarse graining. This is advantageous because it allows to elucidate the different contributions to the interfacial equation arising from the modulations of gap thickness in a systematic way. The local conservation of volume, $\nabla \cdot(b \vec{v})=0$, implies that the resulting fluid interface height will not be a conserved variable. Substitution of Darcy's law into this conservation equation shows also that the pressure field will not be harmonic, in contrast with the case of a Hele-Shaw cell of homogeneous gap thickness.

The boundary conditions at the interface are given by the Young-Laplace pressure jump and the continuity of normal velocity,

$$
p_{2}-p_{1}=\sigma\left[\kappa_{\|}+\frac{2}{b(x, y)}\right], \quad v_{n}=-\frac{b^{2}}{12 \mu} \partial_{n} p_{1},
$$

where $p_{1}, p_{2}$ are the pressures of the displacing liquid and the air phase, respectively, $\kappa_{\|}$the in-plane curvature, and $v_{n}$ the normal component of the two-dimensional velocity field, all of them measured at the interface. $\sigma$ is the interfacial tension between the two fluids, and $\mu$ the dynamic viscosity of the invading liquid, which we have assumed to be perfectly wetting. To complete the problem the boundary condition at the inlet, $v(y \rightarrow-\infty)=V$, must be included.

The hydrodynamic equations for the bulk together with the boundary conditions completely specify the free-boundary problem. Projecting the full dynamics into the interfacial degrees of 
freedom, a nonlocal equation for the interface position, $h(x, t)$, can be derived. All technical details can be found in Refs. [22] and [24]. It is sufficient for our purposes here to keep the lowest-order relevant terms of this equation in Fourier space, to obtain

$$
\frac{1}{V} \frac{\partial \hat{h}(k, t)}{\partial t}=\delta(k)-|k|\left[1+\left(\ell_{1} k\right)^{2}\right] \hat{h}(k, t)+\mathcal{N}_{h}(k)+\frac{1}{2}\left(2-\ell_{2}|k|\right) \hat{\zeta}_{h}(k)+\frac{1}{V} \delta \hat{v}_{\zeta}(k, t),
$$

where $\delta(k)$ is Dirac's delta function with argument the wave vector $k$ of the particular mode considered, accounting for the constant flow rate boundary condition, $\hat{h}(k, t)$ and $\hat{\zeta}_{h}(k)$ are the Fourier transforms of $h(x, t)$ and $\zeta(x, h(x))$, respectively, $\mathcal{N}_{h}(k)$ denotes the leading (quadratic) nonlinearities, and $\delta \hat{v}_{\zeta}(k, t)$ is a bulk term that accounts for the influence of the cell-depth modulations in the region invaded by the displacing liquid. The characteristic lengths $\ell_{1}$ and $\ell_{2}$ are defined as

$$
\ell_{1}=\frac{b_{0}}{\sqrt{\mathrm{Ca}}}, \quad \ell_{2}=\frac{2 b_{0}}{\mathrm{Ca}},
$$

in terms of a capillary number $\mathrm{Ca}=12 \mu V / \sigma$. To make contact with Eq. (6) in Ref. [22] we use that the bulk term $\delta \hat{v}_{\zeta}(k, t)$ decomposes into $\delta \hat{v}_{\zeta}(k, t)=(3 V / 2)\left[-\hat{\zeta}_{h}(k)+\hat{\Omega}_{L R}(k, t)\right]$ [25], where $\hat{\Omega}_{L R}(k, t)$ is a long-ranged correlated contribution given explicitly in Ref. [22]. Equation (4) holds under a weak modulation assumption, meaning that multiplicative terms of order $h \zeta$ or nonlinear terms in $\zeta$ have been neglected. The term in Eq. (4) proportional to $\hat{h}(k, t)$ is well known after the work of Saffman and Taylor [26]. It includes a term in $|k|$, arising from the viscous pressure jump at the interface (a stabilizing term in the present case), and another term in $|k| k^{2}$, associated with the in-plane interfacial tension which stabilizes the interface against deformations. Assuming $|\nabla h| \ll 1$, the leading nonlinear contribution $\mathcal{N}_{h}$ can be neglected. We can also distinguish different terms that contribute to deform the fluid interface in different ways, although they come from the same physical origin, the modulation in gap thickness: a conserved (area-preserving) term proportional to $|k| \hat{\zeta}_{h}$ associated with capillary pressure variations, a nonconserved term proportional to $\hat{\zeta}_{h}$ associated with permeability changes, and, finally, a term proportional to $\delta \hat{v}_{\zeta}$ that accounts for the effect of the gap-thickness modulations in the regions already visited by the interface, hence introducing long-range memory effects [22]. The length $\ell_{1}$ is a crossover length between the stabilizing effects of interfacial tension and viscosity contrast. The length $\ell_{2}$ is a second crossover length, now between the destabilizing effects on the interface of capillary and permeability contributions.

Equation (4) becomes much simpler in the particular case of persistent gap modulation in the direction of front advancement $y$, so that $\zeta=\zeta(x)$ only. In that case $\delta \hat{v}_{\zeta}$ becomes identically zero in the linear approximation, as shown in Refs. [22,24].

The dynamics of the interface under arbitrary variations in gap thickness $\zeta(x)$ can be obtained then by propagating the effect of a localized perturbation. Specifically,

$$
h(x, t)=\int_{\mathbb{R}} d x^{\prime} \zeta\left(x^{\prime}\right) G\left(x-x^{\prime} ; t\right),
$$

where $G\left(x-x^{\prime} ; t\right)$ is the Green's function, and describes how a localized gap inhomogeneity affects the morphology of the invading front. The analytical solution of the propagator in the steady state is obtained by taking $\zeta(x)=\delta(x)$, which leads to $h(x, t)=G(x, t)$. Comparing with the surviving terms in Eq. (4), we obtain

$$
\hat{G}_{s t}(k)=\hat{G}_{s t, c}(k)+\hat{G}_{s t, p}(k)=-\frac{\ell_{2}}{2\left[1+\left(\ell_{1} k\right)^{2}\right]}+\frac{1}{|k|\left[1+\left(\ell_{1} k\right)^{2}\right]}
$$

in Fourier space. This expression shows how each surviving term in Eq. (4) propagates separately. The first and second terms in the right-hand side of Eq. (7) account for the propagation of capillary and permeability effects, respectively. As mentioned above, despite having the same physical origin, the two terms have opposing effects on the deformation of the interface. A locally smaller gap thickness leads simultaneously to a locally lower permeability, which slows downs the front, and a locally higher capillary pressure difference, which pushes the interface harder ahead. 


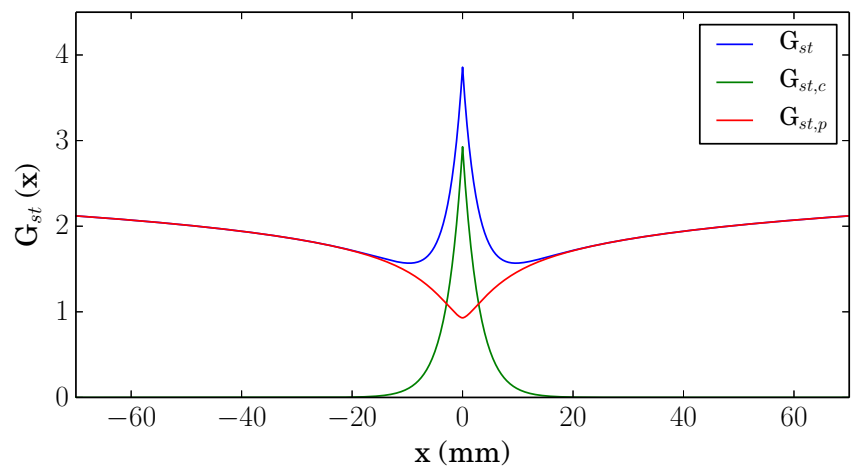

FIG. 2. Steady-state propagator [Eqs. (8), (9), and (10)] computed for $\ell_{1}=2.95 \mathrm{~mm}$ and $\ell_{2}=34.55 \mathrm{~mm}$.

It is also useful to express the Green's function [Eq. (7)] in real space. The result takes the form [27]

$$
G_{s t}(x) \equiv G_{s t, c}(x)+G_{s t, p}(x)
$$

with

$$
G_{s t, c}(x)=\frac{\ell_{2}}{4 \ell_{1}} e^{-|x| / \ell_{1}}
$$

and

$$
G_{s t, p}(x)=\frac{1}{\pi}\left[\ln |x|+\gamma+\ln (2 \pi)-\frac{1}{2}\left\{e^{|x| / \ell_{1}} \mathrm{E}_{\mathrm{i}}\left(-\frac{|x|}{\ell_{1}}\right)+e^{-|x| / \ell_{1}} \mathrm{E}_{\mathrm{i}}\left(\frac{|x|}{\ell_{1}}\right)\right\}\right],
$$

where $\gamma$ is the Euler's constant and $\mathrm{E}_{\mathrm{i}}$ is the exponential-integral function defined as $\mathrm{E}_{i}(x)=$ $-\int_{-x}^{\infty} d t e^{-t} / t$. The propagator $G_{s t, c}(x)$ associated with capillarity has finite range, meaning that capillary effects can only propagate over a finite distance along the interface on the scale of $\ell_{1}$. On the contrary, permeability effects are dominant at large distances from the local disturbance because $G_{s t, p}(x)$ grows logarithmically for $|x| \gg \ell_{1}$. Therefore, a local modulation in gap thickness produces significant deformations of the fluid front in regions very far apart, thus reflecting the nonlocal character of the interface dynamics. Figure 2 shows an example of the propagator in the steady state, and the contribution of the different terms.

In order to compare theory and experiments we introduce an experimentally accessible persistent modulation. It consists of an array of tracks of constant height, $\delta b$, parallel to the direction of fluid advancement, $y$ (Fig. 1). For this specific gap modulation $\zeta(x)$ reads

$$
\zeta(x)=\left[\left(\frac{b_{0}-\delta b}{b_{0}}\right)^{2}-1\right] \sum_{n \in \mathbb{Z}}\left[\theta\left(x-a n+\frac{w}{2}\right)-\theta\left(x-a n-\frac{w}{2}\right)\right],
$$

where $\theta$ is Heaviside step function, $w$ the width of one track, and $a$ the separation between the centerlines of neighboring tracks. The prefactor in Eq. (11) relates the modulation of the Hele-Shaw cell, $\zeta$, to its local gap spacing, $b_{0}$ or $b_{0}-\delta b$, according to Eq. (1).

\section{EXPERIMENTAL SETUP AND COMPUTED FRONT SHAPES}

The experimental setup consists of a Hele-Shaw cell of size $L_{x} \times L_{y}=190 \times 500 \mathrm{~mm}$, made of a top glass plate ( $10 \mathrm{~mm}$ thick) and a bottom fiberglass plate resting on a $15 \mathrm{~mm}$-thick steel platform. The plates are separated by a thin rectangular PVC frame which plays the role of the gap spacer, imposing a well-controlled gap spacing $b_{0}$, and also closes the cell hermetically. 
The gap modulation consists of three equally spaced copper tracks, parallel to the direction of fluid propagation, of height $\delta b=0.06 \pm 0.01 \mathrm{~mm}$ (as determined by interferometric microscopy), width $w=3 \mathrm{~mm}$ and separation $a=48 \mathrm{~mm}$, attached to the bottom plate (Fig. 1), so that the gap takes values $b_{0}$ or $b_{0}-\delta b$. The displacing fluid wets the lateral spacers, thus tending to advance preferentially along the walls, specially for larger gaps. The tracks closer to the two lateral boundaries are intended to screen this effect. Even though the Laplacian character of the pressure field in the absence of gap modulation makes the influence from the boundaries long ranged, we checked in Ref. [28] that this influence has a limited effective penetration length of less than $10 \mathrm{~mm}$ in the range of parameters (gap spacings and average velocities) explored here. For this reason introducing two lateral tracks at a distance of about $50 \mathrm{~mm}$ from the lateral boundaries (much larger than the effective penetration length) seems to be sufficient to screen their influence.

We use silicone oil Rhodorsil $47 \mathrm{~V}$ because of its high wettability, i.e., low contact angle $\theta_{c} \simeq 0^{\circ}$. The oil physical properties are: kinematic viscosity $v=50 \mathrm{~mm}^{2} / \mathrm{s}$, density $\rho=998 \mathrm{~kg} / \mathrm{m}^{3}$ and oil-air surface tension $\sigma=20.7 \mathrm{mN} / \mathrm{m}$ at room temperature. The oil gets into the cell at constant flow rate from one of its short sides, and the resident air leaves the cell at the other side. To obtain smooth injection the oil fills a reservoir before entering the space between the parallel plates. An auxiliary system allows the injection of oil at high pressure in order to obtain initial flat interfaces. We have explored different flow rates corresponding to average front velocities $V$ in the range 0.1 to $0.6 \mathrm{~mm} / \mathrm{s}$ (capillary numbers from $2.9 \times 10^{-3}$ to $17.4 \times 10^{-3}$ ), and different gap thickness $b_{0}$ between 0.26 and $0.66 \mathrm{~mm}( \pm 0.02 \mathrm{~mm})$, represented in Fig. 3 .

A Motion Pro X3 Plus camera, with spatial resolution of $0.19 \mathrm{~mm} /$ pixel, takes pictures of the cell at $30 \mathrm{fps}$. We track the advancing fluid fronts through the experiment, and extract the position of the interfaces by image analysis to obtain the coordinates of the fronts $h(x, t)$ for later analysis. We have used a classical edge detection method. It consists on subtracting a background image from the experimental images to improve contrast and homogeneity, binarizing the gray scale, and resolving the contour between black and white regions. The error on the interface position is around 1 pixel, $0.19 \mathrm{~mm}$, which is typically of the order of half the cell gap thickness. All experiments have been repeated a minimum of five times to ensure reproducibility.

The steady-state interface morphology can be obtained analytically for the particular case of a periodic modulation in gap spacing, corresponding to an infinite array of tracks. In this case $\zeta(x)=\zeta(|x-a|)$, which leads in Fourier space to a discrete set of wave vectors $k=(2 \pi / a) n$, with $n=1,2,3 \ldots$ Taking advantage of the fact that the convolution product of Eq. (6) is an ordinary
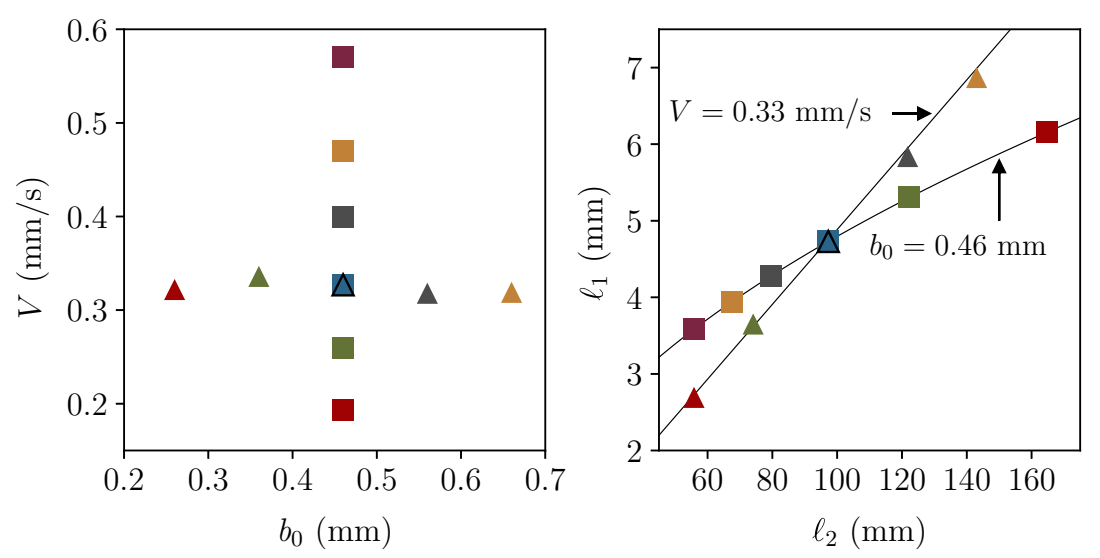

FIG. 3. Parameter space showing the experimental values studied, $b_{0}$ and $V$ (left). Squares correspond to experiments of same gap thickness and triangles to experiments of approximately the same front velocity. Their corresponding values of $\ell_{1}$ and $\ell_{2}$ are shown on the right. The lines are guides to the eye of the form $\ell_{2} \sim \ell_{1}^{2}$ (for $b_{0}$ constant) and $\ell_{2} \sim \ell_{1}$ (for $V$ constant). 
product in Fourier space, $\hat{h}(k)=\hat{G_{s t}}(k) \hat{\zeta}(k)$, and $\hat{\zeta}(k)$ is a sinc function in the case of a rectangular modulation [Eq. (11)], we obtain

$$
\hat{h}(k)=\left[\left(\frac{b_{0}-\delta b}{b_{0}}\right)^{2}-1\right] \frac{2-|k| \ell_{2}}{k|k|\left[1+\left(\ell_{1} k\right)^{2}\right]} \sin \left(\frac{w k}{2}\right)
$$

or, in real space,

$$
h(x)=\left[\left(\frac{b_{0}-\delta b}{b_{0}}\right)^{2}-1\right]\left(\frac{a}{2 \pi^{2} \ell_{1}}\right)^{2} \sum_{n=1}^{\infty}\left\{\frac{\left(a-\pi \ell_{2} n\right) \sin \left(\frac{\pi w n}{a}\right)}{n^{2}\left[\left(\frac{a}{2 \pi \ell_{1}}\right)^{2}+n^{2}\right]} \cos \left(\frac{2 \pi n x}{a}\right)\right\} .
$$

The series convergence is sufficiently fast to reach the experimental resolution with about 40 terms.

\section{RESULTS}

In order to experimentally achieve a steady state we let the fluid front advance for at least half of the total distance of the cell, and we start the measurement after verifying that the morphology is not changing over time. Figure 4 shows the temporal evolution of the front for a particular gap $b_{0}=0.36 \mathrm{~mm}$. The nearly flat lines at the bottom of this figure result from subtracting the front profile at different times, showing that the front morphology remains constant in time.

To study the influence of the gap thickness on the steady-state front morphology, we measured the front spatial profile for different values of $b_{0}$, while keeping the mean velocity of the front constant (Fig. 5). The front profiles obtained experimentally are compared to theoretical predictions obtained numerically from Eq. (13) with no adjustable parameters (Fig. 5). Deviations from the theoretical predictions are predominantly observed at the lateral tails of the front because of the presence of the lateral cell boundaries. However, near the central track our measurements show good agreement with the theoretical results, specially for small gap perturbations [Fig. 6(a)]. Keeping $V$ constant, the deviations from a flat interface increase near the track for increasing the gap perturbation ratio $\delta b / b_{0}$, as expected. For relative changes in the gap thickness above approximately $13 \%$, we observe that theoretical predictions start to deviate from the experimental observation, specially in the area near the modulation, as variations in capillary pressure cause sharp variations in the front morphology. Indeed, as the parameter $b_{0}$ decreases the distortion of the morphology is more localized around the

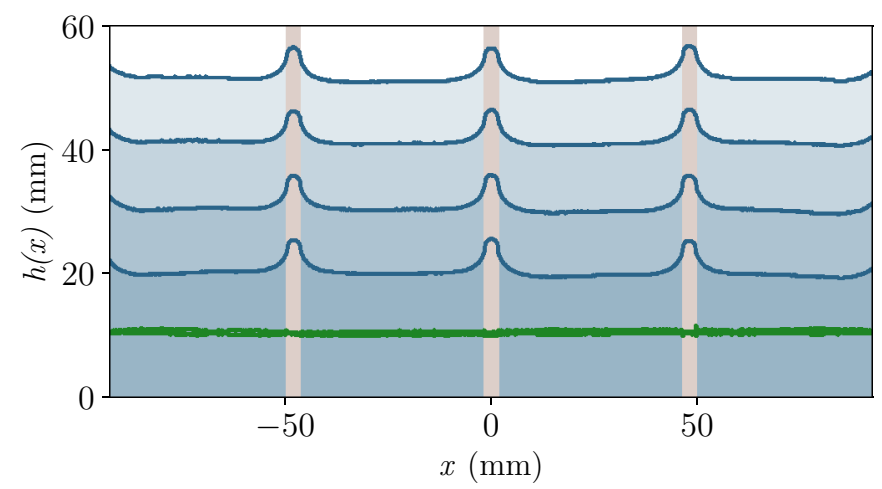

FIG. 4. Morphology of successive fluid fronts and time evolution for a gap thickness $b_{0}=0.36 \mathrm{~mm}$ and average velocity $V=0.33 \mathrm{~mm} / \mathrm{s}$. The vertical narrow stripes represent the regions where the gap modulation is present. Interfaces are plotted at equal time intervals $\Delta t=33.3 \mathrm{~s}$. The green curves at the bottom are differences between successive fronts. In this plot differences between consecutive interfaces have a root mean square deviation smaller than $0.3 \mathrm{~mm}$, which we identify with the steady state. 


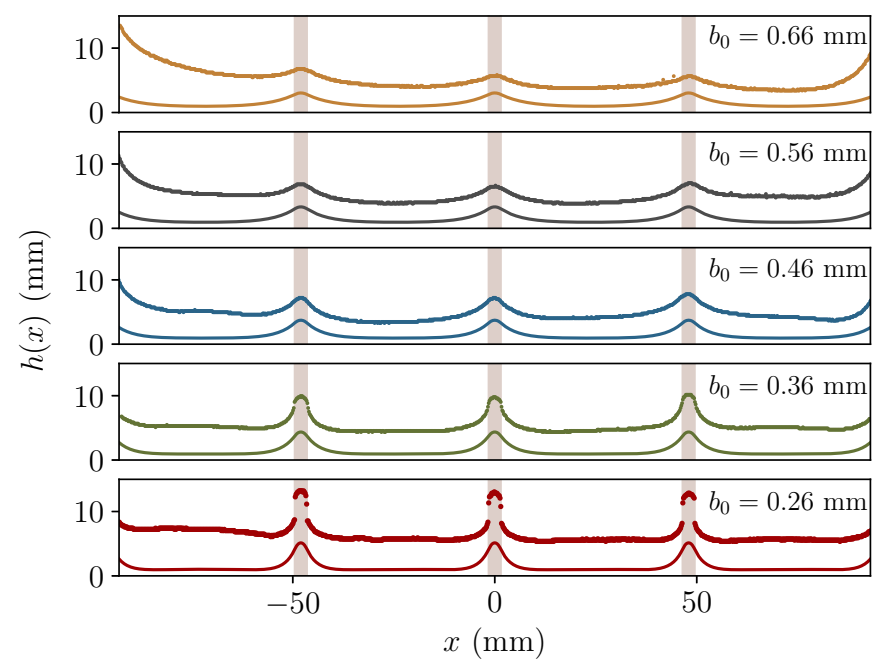

FIG. 5. Steady-state morphology of the fronts for different gap spacings $b_{0}$ and similar average velocity $V=0.33 \pm 0.02 \mathrm{~mm} / \mathrm{s}$. For each value of $b_{0}$ the lower line corresponds to the theoretical prediction and the upper dots to one experimental measurement. Curves have been shifted arbitrarily in the vertical direction for clarity.

heterogeneity. These deviations are expected, as our theoretical description is only valid in the limit $\left|\partial_{x} h\right| \ll 1$.

For a fixed gap thickness $b_{0}=0.46 \mathrm{~mm}$ and, therefore, fixed variation in gap thickness $\left(\delta b / b_{0}=\right.$ 0.13 ), we performed experiments at various values of $V$ [Fig. 6(b)]. Given that viscous forces tend to
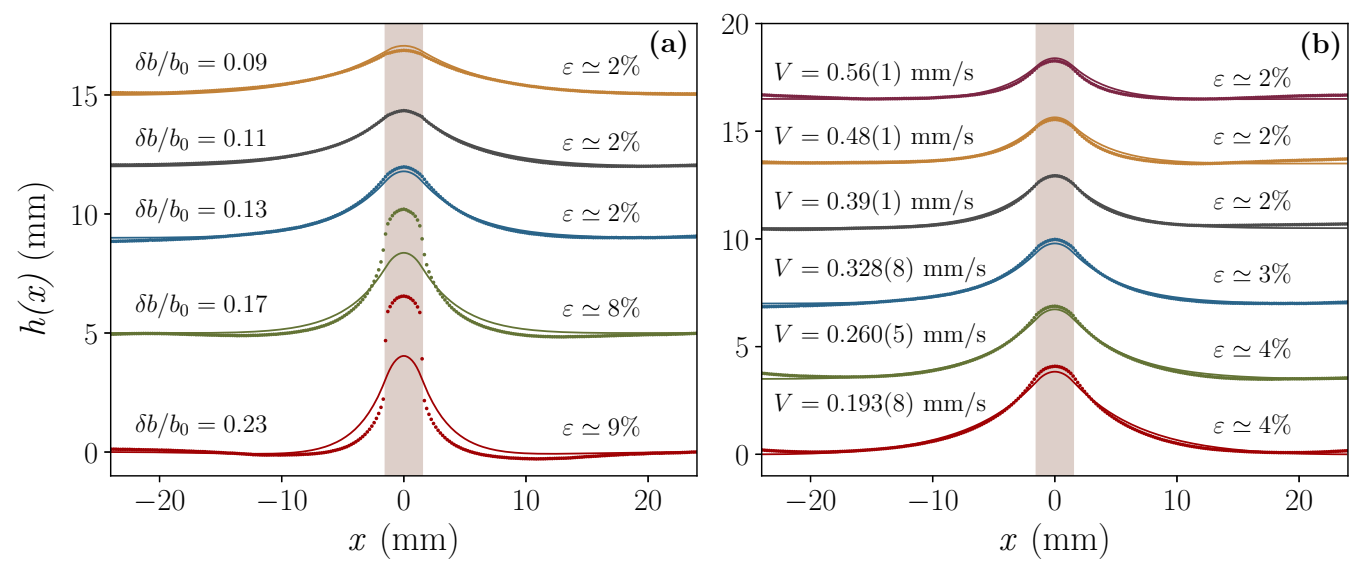

FIG. 6. Steady-state morphology of the fronts around the central track for (a) different gap spacings $b_{0}$ and similar average velocity $V=0.33 \pm 0.02 \mathrm{~mm} / \mathrm{s}$, and (b) different front velocities $V$ and same gap thickness $b_{0}=0.46 \mathrm{~mm}\left(\delta b / b_{0}=0.13\right)$. Continuous lines correspond to the analytical solution and dots to experimental data. Curves have been shifted arbitrarily in the vertical direction for clarity. Each experimental front is obtained from averaging all the steady-state interfaces recorded in 5 to 10 realizations of the same experiment. The root mean square deviation of all the averaged interfaces is of the order of the spatial resolution in the central peak and about $0.5 \mathrm{~mm}$ at the tails. The values of $\varepsilon$ provide the average distance between theoretical and experimental fronts in the range of $x$ shown in the figure, relative to the maximum of the theoretical curve, $h_{\mathrm{M}}$. The analytical curve has been superposed to the experimental data by minimizing the average distance between the two. 

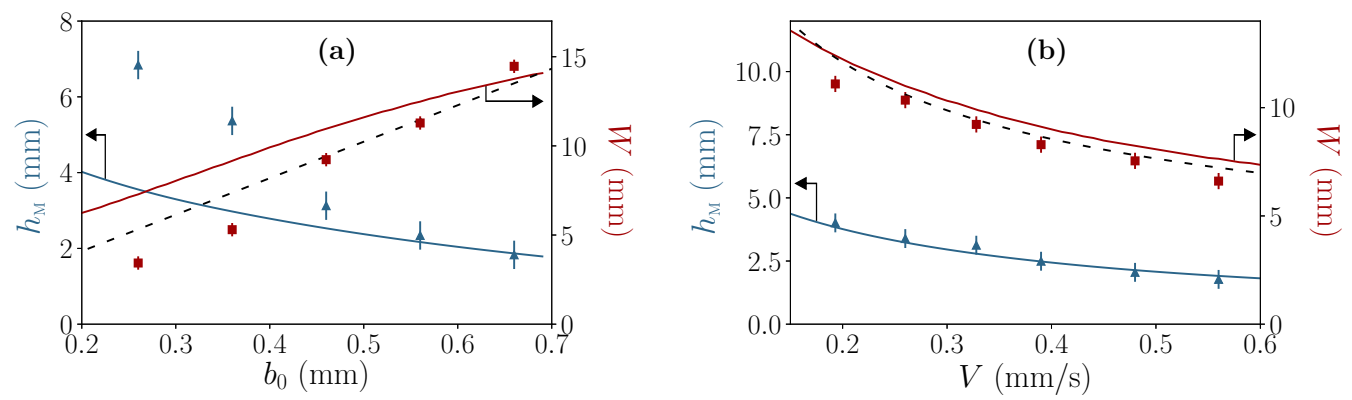

FIG. 7. Maximum distortion of the front height $h_{\mathrm{M}}$ (solid triangles) and front width $W$ at height $h_{\mathrm{M}} / e$ (solid squares) of the fronts shown in Figs. 6(a) and 6(b), respectively. Symbols correspond to experimental measurements and solid lines to the analytical prediction. The characteristic length $2 \ell_{1}$ (dashed line) is also shown for comparison with $W$.

make the interfaces flat, front distortions are more pronounced at lower velocities. In this case the theoretical predictions accurately capture the front morphology for all the range of velocities explored.

In order to further characterize the deviations of the theoretical predictions from the experimental observations, with no adjustable parameters, we focus on the region around the central track $[(-24,24) \mathrm{mm}]$, where the influence from the boundary is negligible, and quantify the deviations of two geometrical quantities, namely the maximum vertical distance $h_{\mathrm{M}}$ and the width of the front $W$ (Fig. 7). $h_{\mathrm{M}}$ is the maximum vertical distance, measured from the most advanced position of the front to the baseline defined by its two tails. $W$ is the width of the front, measured at height $h_{\mathrm{M}} / e$, where $e$ is Napier's constant. There is a good agreement between theory and experiments, especially for small perturbations in gap thickness (i.e., for increasing values of the gap thickness). We also show the characteristic length $2 \ell_{1}$, which compares well with $W$.

In addition we performed experiments with a topography complementary to the one discussed above. In this new geometry copper regions are wide $(w=15 a / 16)$ while fiberglass regions are narrow $(a / 16)$, as shown in Fig. 8. The narrow tracks now retard the front instead of making it advance preferentially, a feature also captured by our theoretical description (Fig. 8), which predicts front morphologies identical to those shown in Fig. 5 for the previous topography but of opposite sign.

\section{DISCUSSION}

The range of validity of our model is defined by two different conditions. First, $|\nabla b| \ll 1$, i.e., gap variations must be sufficiently smooth, and, second, $|\nabla h| \ll 1$ also, ensuring that front modulations are small enough for the linear approximations to apply.

Sharp-edged modulations are not accounted for by the theory, so that their effect can be relevant $a$ priori in the whole range of parameters studied here. Sharp-edged modulations however seem to be innocuous for gap modulations smaller than $13 \%$ of the channel depth, as Fig. 6(a) reveals. The large deviations observed between theory and experiment for larger gap modulations must be attributed to the sharp edges, not to linearization of the equations. This is visible when the deformations of the theoretical front driven at the lowest velocity $[V=0.193(8) \mathrm{mm} / \mathrm{s}]$ in Fig. 6(b) are compared to the deformation of the theoretical front at $\delta b / b_{0}=0.17$ in Fig. 6(a). Both have a comparable maximum $|\nabla h|$, and hence the nonlinear effects should affect them in the same manner, but the agreement with the experimental fronts is clearly much better in the former case than in the latter.

Figure 7(a) corroborates that our theoretical results are in better agreement with the experimental observations for larger $b_{0}$, i.e., for low intensity of the gap modulation, $\delta b / b_{0}$. The influence of $\delta b / b_{0}$ comes into the analytical solution as a prefactor in the linear theory [see Eq. (11)]. The dependence on $b_{0}$ is also contained in $\ell_{1}$ and $\ell_{2}$, the only length scales that appear in the analytical solutions. We have argued, however, that the discrepancies between theory and experiments at small values 


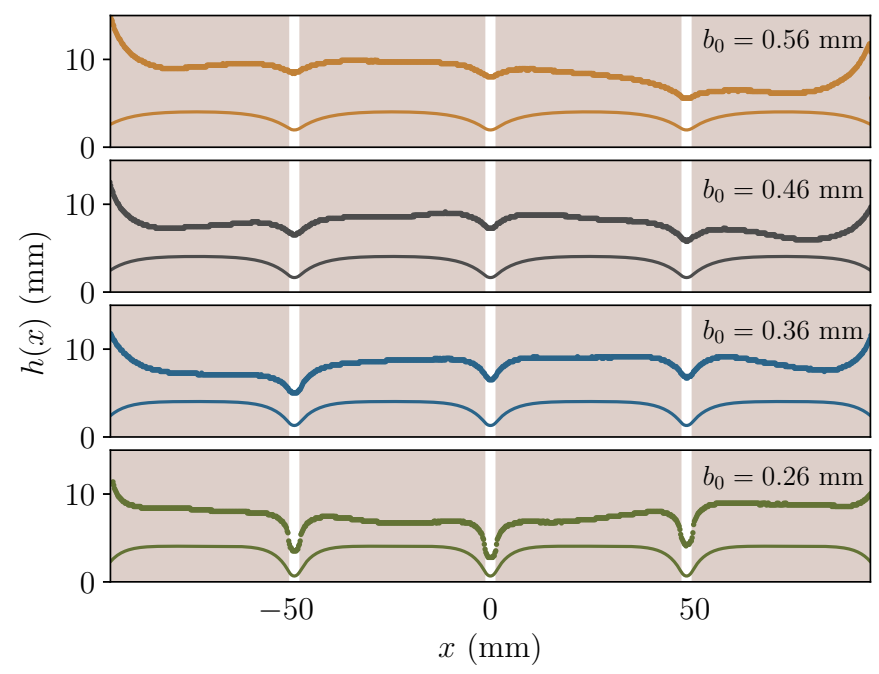

FIG. 8. Examples of the oil-air interface morphology in the case of complementary modulation. The figure shows the steady-state morphology of the fronts for different gap spacings $b_{0}$ and average velocity $V=0.33 \pm 0.02 \mathrm{~mm} / \mathrm{s}$. For each value of $b_{0}$ the lower line corresponds to the theoretical prediction and the upper dots to one experimental measurement. Curves are shifted arbitrarily in the vertical direction for clarity.

of $b_{0}$ originate from the condition $\left|\partial_{x} b\right| \ll 1$ not being fulfilled for too sharp-edged modulations, irrespective of any specific value of either $\ell_{1}$ or $\ell_{2}$. This can be seen also from comparing the experiment of higher $V$ (dark red square) and the one of smaller $b_{0}$ (red triangle) in Fig. 3. Both have a similar $\ell_{2}$, but the analytical curve reproduces satisfactorily the case of large $V$ only, i.e., larger $\ell_{1}$. Furthermore, the experiment of larger $V$ (dark red square) can also be compared to the experiment with the second smaller $b_{0}$ (green triangle), since both of them have similar $\ell_{1}$; in spite of that, Fig. 6 shows that the only one of these two experiments that is in good agreement with the theoretical prediction is the experiment of larger velocity $(V=0.56 \mathrm{~mm} / \mathrm{s})$ and gap spacing $b_{0}=0.46 \mathrm{~mm}$ (the dark red square in Fig. 3). In summary, increasing the intensity of the modulation (making $b_{0}$ small) seems to introduce nonlinear effects associated to $\delta b / b_{0}$ that are not captured by the linear model.

For the model to be valid, the condition $|\nabla h| \ll 1$ should also be satisfied. This will depend on viscosity and surface tension of the invading fluid. In our particular case the model applies well down to velocities as low as $0.193(8) \mathrm{mm} / \mathrm{s}$ for which $|\nabla h| \simeq 0.8$ at the inflection point of the front profile, for a gap thickness of $0.46 \mathrm{~mm}$.

Interestingly, the values of $W$ in Fig. 7 are very similar to the corresponding values of $2 \ell_{1}$ in the regime where the present theory is valid. This is a natural consequence of the fact that $\ell_{1}$ (the crossover length scale between surface tension damping of short-wavelength perturbations and viscous damping of long-wavelength perturbations) coincides with the correlation length along the interface [16].

Due to experimental limitations we cannot explore the regime where permeability effects are comparable in importance to capillary effects. This would require values of $\ell_{2}$ similar to or smaller than half the distance between gap modulations, which amounts to capillary numbers one order of magnitude larger than the capillary numbers explored in the present investigation.

Since our geometry exhibits mirror symmetry in the flow direction with respect to the central track, and the displacement is Saffman-Taylor stable, we expect the interface to be symmetric also. This has proved hard to achieve since small perturbations can easily produce distortions of the front. For example, the displacements shown in Fig. 8 for the case where the gap modulation consists of transverse grooves (complementary modulation) display indeed small dissymmetries, particularly at the largest gap thickness. Interfacial displacements in horizontal Hele-Shaw cells are known to be very sensitive to slight disturbances. Indeed, lateral symmetry breaking of the Saffman-Taylor 
finger and a propagating bubble in the presence of depth modulations transverse to the flow have been reported in unstable displacements in Refs. [12,29]. While it has been known for a long time that the Saffman-Taylor viscous finger is prone to undergo secondary instabilities in the presence of disturbances (see, e.g., Ref. [11] for a review), no such instabilities have been reported so far for stable fronts in Hele-Shaw cells to the best of our knowledge.

\section{CONCLUSIONS}

We have experimentally studied a stable two-phase displacement in which a viscous wetting fluid (silicone oil) displaces a less viscous nonwetting fluid (air), in a Hele-Shaw cell with a periodic gap modulation along $x$ transverse to the direction of fluid front propagation. We have derived theoretically the steady-state front profile in the linear approximation, $\left|\partial_{x} h\right| \ll 1$, and for smooth gap modulations, $\left|\partial_{x} b\right| \ll 1$, specifically for periodic modulations in gap thickness. Because the presence of lateral walls of the cell is not accounted for by the theory, the introduction of a periodic gap modulation is justified in order to screen the effect of the lateral walls, and to have more control on the penetration of the influence of the side boundaries.

Experimental results and theoretical predictions in the steady state show good agreement, with no adjustable parameters, for various intensities of the gap modulation $\delta b / b_{0}$ and front velocities $V$. The agreement is particularly good for small perturbations in gap thickness (below approximately $\delta b / b_{0} \simeq 0.13$ ), for which the theoretical approximations hold.

These results can help understand the influence of topography on interfacial flows, in microfluidic devices in particular $[23,30]$.

\section{ACKNOWLEDGMENTS}

We acknowledge fruitful discussions with Jordi Soriano, Xavier Clotet, and Stéphane Santucci. R.P. is supported by the Secretary for Universities and Research of the Ministry of Economy and Knowledge of the Government of Catalonia (Spain) and the COFUND programme of the Marie Curie Actions of the 7th R\&D Framework Programme of the European Union (Contract No. 2014 BP-B 00138). This work is supported by AGAUR (Generalitat de Catalunya) and MINECO (Spain) through Projects No. 2014 SGR 878 and No. FIS2016-78507-C2-2-P, respectively.

[1] M. C. Cross and P. C. Hohenberg, Pattern formation outside of equilibrium, Rev. Mod. Phys. 65, 851 (1993).

[2] A. D. Stroock and G. M. Whitesides, Controlling flows in microchannels with patterned surface charge and topography, Accounts Chem. Res. 36, 597 (2003).

[3] C. Chung, M. Lee, K. Char, K. H. Ahn, and S. J. Lee, Droplet dynamics passing through obstructions in confined microchannel flow, Microfluid Nanofluidics 9, 1151 (2010).

[4] S. Protière, M. Z. Bazant, D. A. Weitz, and H. A. Stone, Droplet breakup in flow past an obstacle: A capillary instability due to permeability variations, EPL 92, 54002 (2010).

[5] J. Soriano, J. J. Ramasco, M. A. Rodríguez, A. Hernández-Machado, and J. Ortín, Anomalous Roughening of Hele-Shaw Flows with Quenched Disorder, Phys. Rev. Lett. 89, 026102 (2002).

[6] D. Geromichalos, F. Mugele, and S. Herminghaus, Nonlocal Dynamics of Spontaneous Imbibition Fronts, Phys. Rev. Lett. 89, 104503 (2002).

[7] J. Casademunt, Viscous fingering as a paradigm of interfacial pattern formation: Recent results and new challenges, Chaos 14, 809 (2004).

[8] H. Zhao, J. Casademunt, C. Yeung, and J. V. Maher, Perturbing Hele-Shaw flow with a small gap gradient, Phys. Rev. A 45, 2455 (1992). 


\section{LAUTARO DÍAZ-PIOLA et al.}

[9] T. T. Al-Housseiny, P. A. Tsai, and H. A. Stone, Control of interfacial instabilities using flow geometry, Nat. Phys. 8, 747 (2012).

[10] T. T. Al-Housseiny and H. A. Stone, Controlling viscous fingering in tapered Hele-Shaw cells, Phys. Fluids 25, 092102 (2013).

[11] K.V. McCloud and J.V. Maher, Experimental perturbations to Saffman-Taylor flow, Phys. Rep. 260, 139 (1995).

[12] A. Franco-Gómez, A. B. Thompson, A. L. Hazel, and A. Juel, Sensitivity of Saffman-Taylor fingers to channel-depth perturbations, J. Fluid Mech. 794, 343 (2016).

[13] M. A. Rubio, C. A. Edwards, A. Dougherty, and J. P. Gollub, Self-Affine Fractal Interfaces From Immiscible Displacement in Porous Media, Phys. Rev. Lett. 63, 1685 (1989).

[14] A. Dougherty and N. Carle, Distribution of avalanches in interfacial motion in a porous medium, Phys. Rev. E 58, 2889 (1998).

[15] J. Soriano, A. Mercier, R. Planet, A. Hernández-Machado, M. A. Rodríguez, and J. Ortín, Anomalous Roughening of Viscous Fluid Fronts in Spontaneous Imbibition, Phys. Rev. Lett. 95, 104501 (2005).

[16] M. Alava, M. Dubé, and M. Rost, Imbibition in disordered media, Adv. Phys. 53, 83 (2004).

[17] R. Planet, S. Santucci, and J. Ortín, Avalanches and Non-Gaussian Fluctuations of the Global Velocity of Imbibition Fronts, Phys. Rev. Lett. 102, 094502 (2009).

[18] X. Clotet, J. Ortín, and S. Santucci, Disorder-Induced Capillary Bursts Control Intermittency in Slow Imbibition, Phys. Rev. Lett. 113, 074501 (2014).

[19] P.-G. de Gennes, Imperfect Hele-Shaw cells, J. Physique (Paris) 47, 1541 (1986).

[20] V. Ganesan and H. Brenner, Dynamics of Two-Phase Fluid Interfaces in Random Porous Media, Phys. Rev. Lett. 81, 578 (1998).

[21] M. Dubé, M. Rost, K. R. Elder, M. Alava, S. Majaniemi, and T. Ala-Nissila, Liquid Conservation and Nonlocal Interface Dynamics in Imbibition, Phys. Rev. Lett. 83, 1628 (1999).

[22] E. Pauné and J. Casademunt, Kinetic Roughening in Two-Phase Fluid Flows Through a Random Hele-Shaw Cell, Phys. Rev. Lett. 90, 144504 (2003).

[23] J. Atencia and D. J. Beebe, Controlled microfluidic interfaces, Nature (London) 437, 648 (2005).

[24] E. Pauné, Interface dynamics in two-dimensional viscous flows, Ph.D. thesis, Universitat de Barcelona (2002), available at http://hdl.handle.net/2445/35490.

[25] Notice that a factor $3 / 2$ in front of the term $\hat{\Omega}_{L R}(k, t)$ is missing in Eq. (6) of Ref. [22], while it is correctly given in Section 6.2 of Ref. [24] .

[26] P. G. Saffman and G. Taylor, The penetration of a fluid into a porous medium or Hele-Shaw cell containing a more viscous liquid, Proc. R. Soc. A 245, 312 (1958).

[27] J. I. Richards and H. K. Youn, Theory of Distributions: A Nontechnical Introduction (Cambridge University Press, Cambridge, 1990).

[28] J. Soriano, J. Ortín, and A. Hernández-Machado, Anomalous roughening in experiments of interfaces in Hele-Shaw flows with strong quenched disorder, Phys. Rev. E 67, 056308 (2003).

[29] A. de Lózar, A. Heap, F. Box, A. L. Hazel, and A. Juel, Tube geometry can force switchlike transitions in the behavior of propagating bubbles, Phys. Fluids 21, 101702 (2009).

[30] S. A. Setu, R. P. A. Dullens, A. Hernández-Machado, I. Pagonabarraga, D. G. A. L. Aarts, and R. Ledesma-Aguilar, Superconfinement tailors fluid flow at microscales, Nat. Commun. 6, 7297 (2015). 